\title{
EVALUACIÓN DEL CRECIMIENTO DE PLANTACIONES JUVENILES DE ALGARROBO BLANCO (Prosopis Alba Griseb) EN EL CENTRO OESTE DEL CHACO ARGENTINO
}

\section{EVALUATION OF THE GROWTH OF YOUNG PLANTATION OF MESQUITE (Prosopis Alba Griseb) IN THE MIDDLE WEST ARGENTINE CHACO}

\author{
Julio Félix Michela*1, Sebastián M. Kees², Juan J. Skoko³
}

Recibido para publicación: Agosto 29 de 2014 - Aceptado para publicación: Mayo 11 de 2015

\section{RESUMEN}

Prosopis alba es la principal especie nativa empleada en la industria del mueble en la provincia del Chaco, Argentina. Sin embargo, a pesar de su importancia económica, no se encuentran antecedentes dasométricos de la especie en la zona. El objetivo del presente trabajo fue investigar el crecimiento de plantaciones jóvenes de algarrobo en la provincia del Chaco, Argentina. Se utilizaron plantaciones de 4, 5 y 7 años de edad con distancias de siembra diferentes. En cada plantación se midió el diámetro normal y la altura total; se calcularon los incrementos medios anuales y las medias aritméticas de éstos en el diámetro y la altura a intérvalos de confianza del 95\%. Además, se comparó el incremento medio anual para el diámetro y la altura de las tres plantaciones mediante un análisis de varianza y se utilizó el test de Tukey para analizar los resultados. En adicción, se calculó la media ponderada de los incrementos medios anuales en diámetro normal y altura. En plantaciones de 7 años de edad, los umbrales inferiores del incremento medio anual en diámetro y altura fueron de 0,158 y $0,80 \mathrm{~m}$ respectivamente; mientras que los umbrales superiores en diámetro y altura fueron de 0,187 y $0,93 \mathrm{~m}$ respectivamente. Las plantaciones de 4 y 5 años edad presentaron incrementos anuales en diámetro significativamente superiores a la plantación de 7 años de edad. La media ponderada de los crecimientos en diámetro y altura fue de 0,172 y $0,87 \mathrm{~m}$ respectivamente

Palabras clave: Forestal, plantación, Parque chaqueño

\begin{abstract}
Prosopis alba is the main native species used in the furniture industry in the el Chaco province, Argentina. Despite the economic importance there are not dasometric records in the Chaco province. The objective of this study was to investigate the growth of P. alba plantations. Three plantations $(4,5$ and 7 years old) at different plant densities were studied.In each plantation, the normal diameter and total height was measured. In addition, the average of annual increases in diameter and height; the arithmetic means of annual increments

\footnotetext{
${ }^{*}$ Ingeniero Forestal. Programa Cambio Rural. Ministerio de Agricultura, Ganadería y Pesca de la Nación. Chaco. Argentina, Teléfono/fax: 54 (0364) 443 8101; e-mail: juliofmichela@hotmail.com; Direccion Jujuy 850 (4200) Santiago del Estero - Argentina.

${ }^{2}$ Ingeniero Forestal. Instituto Nacional de Tecnología Agropecuaria. Argentina. ; Direccion Antartida Argentina, manzana 3, parcela 12 (3536) Presidencia de la Plaza - Chaco,Argentina.

${ }^{3}$ Promotor del programa Cambio Rural. Instituto Nacional de Tecnología Agropecuaria; San Martín 790(3700) Presidencia Roque Sáenz Peña; Chaco,Argentina.
} 
and the weighted mean of annual increases in the average of diameter and height were calculated for each plantation at $95 \%$ confidence level. The analysis of varianza and the Tukey's test were performed to analyze the data output. In seven years old plants, the lower thresholds of the average annual increase in diameter and height were 0,158 and 0,80m, respectively; while the upper thresholds were 0,187 and 0,93m, respectively. Significant differences were observed in plantations of 4 and 5 years old compared to plantations of 7 years old. The weighted average of the growth in diameter and height were 0,172 and 0,87m, respectively.

Key words: Forestry, plantation, Parque chaqueño

\section{INTRODUCCIÓN}

La especie Prosopis alba (Fabaceae, Mimosoideae) identificada comúnmente como algarrobo blanco, es el árbol por antonomasia para los pobladores del Chaco argentino, a los que proporciona sombra, alimento, bebida, combustible y madera de hasta $12 \mathrm{~m}$ de altura y $0,7 \mathrm{~m}$ de diámetro, produce de 5 a $40 \mathrm{~kg}$ de frutos por año y fructifica entre diciembre $y$ marzo (Prokopiuk et al. 2000). En la provincia del Chaco, existe el eje de la fabricación del mueble. Cuadra (2012) indica que, desde 1980 se constituyeron unos incipientes aserraderos orientados a la fabricación de muebles en la ciudad de Machagai, que en pocos años concentró una gran cantidad de instalaciones que abastecía la demanda, no sólo regional, sino nacional. En 2008 se computaron 230 fábricas en dicha localidad y ya se sumaban sus vecinas Quitilipi (orientada a la producción de aberturas) y Presidencia de la Plaza (productos varios), sumando entre las tres alrededor de 450 establecimientos foresto-industriales. Aunque es en Machagai donde se localiza el 55\% de las carpinterías del total provincial. Según estudios realizados por el Ministerio de Economía, Industria y Empleo del Gobierno de la provincia del Chaco (2009) sobre estas mismas fábricas, siguiendo criterios clasificatorios propios, el 80\% de ellas son consideradas medianas o pequeñas. El establecimiento de pequeña dimensión que consume menos de 60 toneladas mensuales con una media de 5 operarios (Cuadra 2011), trabaja de manera artesanal y empleando mano de obra familiar, aunque otros fueron incorporando tecnología y actualmente fabrican tableros a partir de escuadrías que normalmente se destina a la producción de energía. Esta industria del mueble demandó, solo en el año 2012, 52.470 toneladas de rollos según lo publicado por el blog Dirección de bosques de la provincia del Chaco.

Es posible identificar dos programas de incentivos de forestación aplicados en la provincia. Uno de ellos es el Plan Provincial de Expansión Forestal vigente a partir del año 1991. Dentro de este esquema, el Instituto de Investigaciones Forestales y Agropecuarias del Gobierno de la Provincia del Chaco está ejecutando un programa de reforestación de Prosopis alba con fines maderables habiendo plantado 2382 ha entre los años 1993 y 1998 (Prokopiuk et al. 2000, citando a la Subsecretaria de Recursos Naturales y Medio Ambiente 1999). El restante programa se implementa a partir del año 2000 hasta la fecha y está representado por los créditos no reintegrables previstos por la ley 25.080 (de Inversiones para Bosques Cultivados) y su modificatoria la ley 26.432, (Dirección de Producción Forestal. Ministerio de Agricultura, Ganadería y Pesca. Presidencia de la Nación). En el marco de ambos programas se citan plantaciones con algarrobo a partir del año 1993 (Lifton y Aguilar 2007) y según la misma 
fuente en el período comprendido entre ese año y el 2005 se plantaron aproximadamente 9.000 hectáreas. El algarrobo es la principal especie nativa empleada en la industria del mueble en la provincia y a pesar de la importancia económica y de la superficie plantada no se encontraron antecedentes de datos dasométricos elementales de forestaciones de algarrobo para la región, siendo que los estudios de crecimiento de árboles y masas forestales son una herramienta de gran utilidad en la correcta toma de decisiones sobre tratamientos silvícolas y aprovechamientos de los bosques, ya que además facilitan la cuantificación de las respuestas (García et al. 1992). El mismo autor afirma que uno de los componentes del rendimiento de mayor interés es el diámetro promedio. En consecuencia, los resultados de investigar acerca de los incrementos anuales en diámetro y altura de las plantaciones juveniles de algarrobo brindarán una primera aproximación para colaborar con técnicos y plantadores a los efectos de determinar pautas de planificación de manejo de plantaciones de esta especie en el centro oeste de la provincia del Chaco. Los objetivos del presente artículo fueron determinar el incremento medio anual en diámetro y altura de tres plantaciones juveniles de edades diferentes de Prosopis alba; comparar el incremento medio anual en diámetro $y$ altura entre las tres plantaciones y ponderar el incremento medio anual de las tres plantaciones estudiadas.

\section{MATERIALES Y MÉTODOS}

El estudio se realizó en el centro oeste de la provincia del Chaco en una zona aledaña a las localidades de Presidencia Roque Sáenz Peña, Avia terai, Concepción del bermejo y Pampa del infierno, en un rectángulo cuya precisión geográfica se detalla en la figura 1.
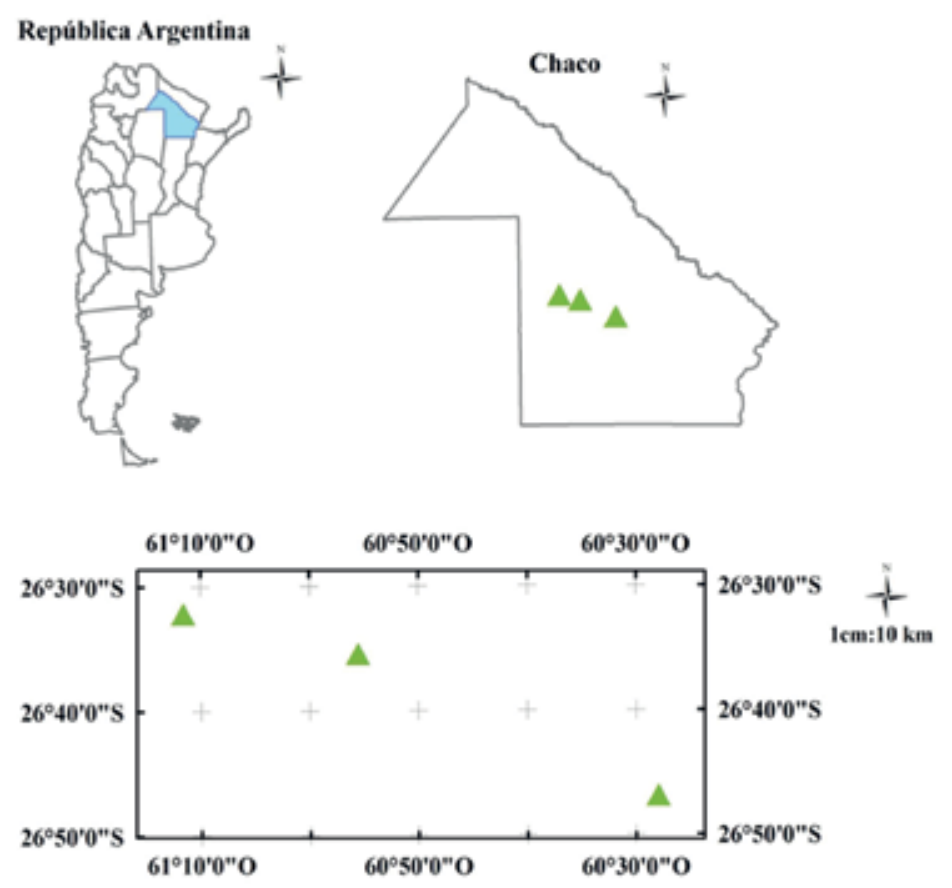

Figura 1. Ubicación de las plantaciones relevadas de $P$. alba en Chaco, Argentina 
El material originario de los suelos del área donde están localizadas las plantaciones estudiadas corresponde a loess y loess-aluvial local fósil (Ledesma et al. 1973). De acuerdo a la clasificación de zonas climáticas del Chaco argentino según el sistema de Köppen el área de estudio está enclavada dentro del subtipo climático cfw'a(h), que corresponde a mesotermal húmedo (Morello y Adámoli 1974).

Se estudiaron tres plantaciones de algarrobo de 4, 5 y 7 años de edad de 4, 0,5 y 14 ha de superficie respectivamente. Se estableció que cada edad corresponde a un tratamiento. Como la plantación de 5 años de edad es un macizo de 0,5 ha de superficie $(50 \times 100)$ se decidió prescindir del muestreo y mensurar todos los ejemplares tomando la precaución de dejar una fila de borde alrededor. En las dos plantaciones restantes se seleccionó aleatoriamente el punto de inicio del área a relevar haciéndolo coincidir con un ejemplar y a partir de este se midieron superficies equivalentes al macizo de 5 años de edad, dejando nuevamente una fila alrededor para que cumpla la función de borde. Dentro de las áreas de medición determinadas en cada plantación se midió el diámetro a 1,3 m respecto al suelo (DAP) y la altura total (HT), es decir entre la superficie del suelo y el respectivo ápice.

Las plantaciones de 4 y 5 años de edad tuvieron una densidad inicial de 400 ejemplares por hectarea y la plantación de 7 años de edad de 625 arboles, con distanciamientos de $5 \times 5$ en las dos primeras y de $4 \times 4$ en la restante. La plantación de 4 años de edad fue podada en una ocasión, mientras que las otras dos fueron podadas en dos oportunidades. En todos los casos los plantines para realizar las plantaciones fueron obtenidos desde el vivero del Instituto de Investigaciones Forestales y Agropecuarias de la provincia del Chaco.
Con los datos resultantes de las mediciones realizadas se calcularon para cada plantación las siguientes variables: incremento medio anual en diámetro (IMAD) e incremento medio anual en altura (IMAH). El valor del crecimiento o incremento medio anual (IMA) enuncia la media del crecimiento total a cierta edad del árbol. Expresa por lo tanto la media anual del crecimiento para cualquier edad (Imaña y Encinas 2008). Se calcularon las medias aritméticas de los incrementos medios anuales y se determinaron los intervalos de confianza con un 95\% de confianza. Se hizo una comparación del incremento medio anual para el diámetro y la altura de las tres plantaciones medidas mediante un análisis de la varianza. El test de Tukey fue la opción para comparar las medias entre tratamientos en caso de que se obtuvieran diferencias significativas entre ellos. Se realizaron las tablas de frecuencias del IMAD e IMAH y sumando los resultados de la relación por producto de las marcas medias de clase con las frecuencias relativas de cada clase, fue posible calcular la media ponderada del IMAD e IMAH del área estudiada. Se empleó el programa InfoStat 2013 (Di Rienzo et al. 2013) versión libre para la sistematización y procesamientos de los datos.

\section{RESULTADOS Y DISCUSIÓN}

En la tabla 1 se muestran los resultados de los cálculos de los intervalos de confianza para el promedio de los IMAD e IMAH con una probabilidad de error del 5\%, se consigna el error experimental (EE), el tamaño de la muestra (n) y los límites de confianza inferior (LI) y superior (LS). 
Michela et al. - Crecimiento de plantaciones juveniles de algarrobo

Tabla 1. Límites de confianza de los incrementos medios anuales de las variables medidas

\begin{tabular}{cccccccc}
\hline Tratamiento & Edad en años & Variable & $\begin{array}{c}\text { Estimación de la } \\
\text { media }\end{array}$ & EE & $\mathbf{n}$ & LI (95\%) & LS (95\%) \\
\hline $\mathbf{1}$ & $\mathbf{4}$ & IMAD & $\mathbf{1 , 8 0}$ & 0,04 & 163 & 1,72 & 1,87 \\
$\mathbf{2}$ & $\mathbf{5}$ & IMAH & $\mathbf{0 , 9 5}$ & 0,01 & 163 & 0,93 & 0,97 \\
$\mathbf{3}$ & $\mathbf{7}$ & IMAD & $\mathbf{1 , 7 7}$ & 0,03 & 102 & 1,71 & 1,84 \\
& & IMAH & $\mathbf{0 , 8 5}$ & 0,01 & 102 & 0,84 & 0,87 \\
& & IMAD & $\mathbf{1 , 6 5}$ & 0,03 & 162 & 1,58 & 1,71 \\
& & IMAH & $\mathbf{0 , 8 2}$ & 0,01 & 162 & 0,80 & 0,84 \\
\hline
\end{tabular}

Las medias aritméticas de los incrementos medios anuales en diámetro y altura son levemente decrecientes a medida que aumentan las edades de las plantaciones. A fin de lograr resultados estadísticamente confiables, se compararon incrementos medios de ambas variables mediante el análisis de la variancia (ANOVA).

Los resultados del ANOVA del IMAD se presentan en la tabla 2, los valores muestran diferencias significativas entre los tratamientos, en consecuencia se empleó el test de Tukey (alfa igual a 0.05) para determinar las diferencias entre las medias. Los IMAD resultaron ser iguales en las dos plantaciones de menor edad y diferentes de estas dos con respecto a la restante (Tabla 3). En la tabla 4 se presentan los resultados del ANOVA para el IMAH, nuevamente se encontraron diferencias significativas entre los tratamientos, en este caso la comparación entre medias empleando el test de Tukey indica que las tres plantaciones presentan IMAH distintos con una certeza del 95\% (Tabla 5), es decir que las tres plantaciones crecieron en altura a tasas diferentes en los períodos considerados.

En la tabla 6 se agrupan los valores correspondientes a los IMAD e IMAH de las tres plantaciones relevadas según clase diamétrica (CD) y clase de altura $(\mathrm{CH})$, límite inferior (LI) y superior de clase (LS), marca media de clase (MC), frecuencia acumulada (FA) y frecuencia relativa (FR) y el cálculo de la media aritmética ponderada (MP).

En el ámbito forestal el crecimiento es considerado como una función que depende de la especie, edad, densidad y calidad de sitio (Rojo Martínez 2005). Si bien Patiño (1995) expresa que de acuerdo con la teoría silvícola del crecimiento, el espaciamiento tiene en promedio, mayor influencia en el desarrollo en diámetro que en el crecimiento en altura de los árboles, García et al. (1992) argumenta que la altura promedio es sensible a la edad, densidad y clase de sitio. En virtud de estas argumentaciones no es posible adjudicar tasas de crecimientos diferentes de las dos variables consideradas solo al paso del tiempo, especialmente en lapsos de tiempos relativamente tan cortos como los siete años considerados. Esta apreciación parece estar en consonancia con los resultados que se observan en el ANOVA de los IMAD, en los cuales se muestra que es el espaciamiento el factor que juega un rol preponderante al momento de provocar tasas de crecimientos en diámetro diferentes. 
TEMAS AGRARIOS - Vol. 20:(1) Enero - Junio 2015 (9 - 18)

Tabla 2. Análisis de la variancia correspondiente al IMAD

\begin{tabular}{lccccc}
\hline $\begin{array}{l}\text { Fuente de } \\
\text { variación }\end{array}$ & $\begin{array}{c}\text { Suma de } \\
\text { cuadrados }\end{array}$ & $\begin{array}{c}\text { Grados de } \\
\text { libertad }\end{array}$ & $\begin{array}{c}\text { Cuadrado } \\
\text { medio }\end{array}$ & F & p-valor \\
\hline Modelo & $\mathbf{1 , 9 9}$ & $\mathbf{2}$ & $\mathbf{1 , 0 0}$ & 5,48 & 0,0045 \\
Tratamiento & $\mathbf{1 , 9 9}$ & $\mathbf{2}$ & $\mathbf{1 , 0 0}$ & 5,48 & 0,0045 \\
Error & $\mathbf{7 7 , 0 6}$ & $\mathbf{4 2 4}$ & $\mathbf{0 , 1 8}$ & & \\
Total & $\mathbf{7 9 , 0 5}$ & $\mathbf{4 2 6}$ & & & \\
\hline
\end{tabular}

Tabla 3. Comparación de medias de IMAD entre los tres tratamientos

\begin{tabular}{ccc}
\hline Tratamiento & Edad en años & $\begin{array}{c}\text { Media } \\
\text { aritmética }\end{array}$ \\
\hline 1 & 4,00 & $1,80 \mathrm{a}$ \\
2 & 5,00 & $1,77 \mathrm{a}$ \\
3 & 7,00 & $1,65 \mathrm{~b}$ \\
\hline
\end{tabular}

Tabla 4. Análisis de la variancia correspondiente al IMAH

\begin{tabular}{lccccc}
\hline $\begin{array}{c}\text { Fuente de } \\
\text { variación }\end{array}$ & $\begin{array}{c}\text { Suma de } \\
\text { cuadrados }\end{array}$ & $\begin{array}{c}\text { Grados de } \\
\text { libertad }\end{array}$ & $\begin{array}{c}\text { Cuadrado } \\
\text { medio }\end{array}$ & F & p-valor \\
\hline Modelo & $\mathbf{1 , 5 8}$ & $\mathbf{2}$ & $\mathbf{0 , 7 9}$ & 50,48 & 0,0001 \\
Tratamiento & $\mathbf{1 , 5 8}$ & $\mathbf{2}$ & $\mathbf{0 , 7 9}$ & 50,48 & 0,0001 \\
Error & $\mathbf{6 , 6 5}$ & $\mathbf{4 2 4}$ & $\mathbf{0 , 2 0}$ & & \\
Total & $\mathbf{8 , 2 3}$ & $\mathbf{4 2 6}$ & & & \\
\hline
\end{tabular}

Tabla 5. Comparación de medias de IMAH entre los tres tratamientos

\begin{tabular}{ccc}
\hline Tratamiento & Edad en años & $\begin{array}{c}\text { Media } \\
\text { aritmética }\end{array}$ \\
\hline 1 & 4,00 & $0,95 a$ \\
2 & 5,00 & $0,85 b$ \\
3 & 7,00 & $0,82 c$ \\
\hline
\end{tabular}

Un escenario distinto se plantea cuando se analiza el crecimiento en altura. Si bien a mayor edad las alturas promedios de los rodales fueron superiores (Tabla 1), todos estos crecieron a tasas diferentes según los resultados del ANOVA de los IMAH. Así, Arteaga y Castelán (2008) citando a Fierros (2001) afirman que la densidad influye en el crecimiento en diámetro, en la forma de los fustes, y en el crecimiento en altura en menor grado. También Patiño (1995) opina que existen algunas controversias con relación a los efectos del espaciamiento sobre el crecimiento en altura de los árboles.

Ferrere et al. (2005) citando a varios autores afirman que, en general, el aumento de la densidad de la plantación afecta al crecimiento 
Tabla 6. Distribución de frecuencias de los IMAD e IMAH y media aritmética ponderada de la variable

\begin{tabular}{ccccccc}
\hline CD & LI & LS & MC & FA & FR & MC*FR \\
\hline 1 & 0,57 & 0,88 & 0,72 & 8 & 0,02 & 0,0144 \\
2 & 0,88 & 1,18 & 1,03 & 37 & 0,09 & 0,0927 \\
3 & 1,18 & 1,49 & 1,34 & 71 & 0,17 & 0,2278 \\
4 & 1,49 & 1,8 & 1,64 & 128 & 0,3 & 0,492 \\
5 & 1,8 & 2,1 & 1,95 & 100 & 0,23 & 0,4485 \\
6 & 2,1 & 2,41 & 2,26 & 57 & 0,13 & 0,2938 \\
7 & 2,41 & 2,72 & 2,56 & 21 & 0,05 & 0,128 \\
8 & 2,72 & 3,03 & 2,87 & 5 & 0,01 & 0,0287 \\
MP & & & & & & 1,7259 \\
CH & $\mathbf{L I}$ & $\mathbf{L S}$ & $\mathbf{M C}$ & $\mathbf{F A}$ & $\mathbf{F R}$ & $\mathbf{M C} * \mathbf{F R}$ \\
1 & 0,39 & 0,5 & 0,44 & 3 & 0,01 & 0,0044 \\
2 & 0,5 & 0,62 & 0,56 & 12 & 0,03 & 0,0168 \\
3 & 0,62 & 0,73 & 0,68 & 43 & 0,1 & 0,068 \\
4 & 0,73 & 0,85 & 0,79 & 125 & 0,29 & 0,2291 \\
5 & 0,85 & 0,97 & 0,91 & 132 & 0,31 & 0,2821 \\
6 & 0,97 & 1,08 & 1,03 & 86 & 0,2 & 0,206 \\
7 & 1,08 & 1,2 & 1,14 & 26 & 0,06 & 0,0684 \\
MP & & & & & & 0,8748 \\
\hline
\end{tabular}

individual de los árboles, manifestándo por una disminución en el diámetro y la altura. Esta particularidad se cumple parcialmente en este estudio, puesto que los valores promedios de la variables son decrecientes a medida que disminuye el espaciamiento, pero también con la edad de los rodales. Esto hace presumir que algún otro factor entra en juego, ya sea características específicas del sitio o bien la necesidad de un aclareo en la plantación de mayor edad.

Precisamente Atanasio (2014), al evaluar la influencia de raleos selectivos sobre el crecimiento de $P$. alba en plantaciones en un período de 9 años a 18 años de edad en la provincia del Chaco con un régimen de precipitación media de 1000 mm.año ${ }^{-1}$, afirma que la práctica de raleo permite mejorar la condición de la masa, dado que se eliminan los ejemplares de mala forma y se favorece el crecimiento individual de los árboles en diámetro y altura. Se encontraron similitudes al comparar los crecimientos de las variables estudiadas con otras especies afines. De esta manera, del Castillo y Tarnowski (2006) al investigar el crecimiento de Prosopis chilensis en el Pedemonte de Yungas en la provincia de Jujuy con precipitaciones que oscilan entre 300 y 500 mm anuales hallaron que la 
mayor parte de los ejemplares investigados se encuentran al cuarto año de edad entre los 6 y $11 \mathrm{~cm}$ de DAP. Ribaski y Menezes (2002) aseveran que en la región semiárida brasileña los promedios dendrométricos calculados en sistemas silvopastoriles con Prosopis juliflora de aproximadamente 15 años de edad fueron 7,4 m de altura total del árbol y 25,7 cm de diámetro en la base del fuste. En virtud de las opiniones de los diferentes autores adquiere importancia la posibilidad de poder obtener valores ponderados de los incrementos en diámetro y altura tal como se muestra en la tabla 6, que permitan brindar orientaciones para poder establecer los horizontes de planificación del manejo silvicultural y el aprovechamiento forestal de la especie en la región considerada. Específicamente referido a P. alba, Navall y Senilliani (2004) observaron valores de IMAD e IMAH similares en Santiago del Estero, aunque los diámetros fueron medidos a la altura del cuello. También Pérez et al. (2011) contribuyen con datos concretos al evaluar la calidad de sitio en plantaciones con P. alba en la provincia de Formosa realizadas a partir del año 2005 y definen crecimientos en DAP menores a 1 cm.año-1 en clases provisorias de calidad de sitio calificadas como malas a otros mayores $\mathrm{O}$ iguales a 2,8 cm.año-1, en clases provisorias de calidades de sitios calificadas como excelentes, mientras que afirman que la variación en crecimiento en HT varía para las mismas clases provisorias de calidades de sitio entre menores a 0,70 m.año-1 y mayores o iguales a 1,15 metros. $a_{n} o^{-1}$. Por su parte, Vicentini y Pérez (2011) al evaluar los efectos del suelo en plantaciones forestales de $P$. alba en la provincia de Formosa en latitudes con precipitaciones medias de 1300 mm.año-1 en plantaciones de 4 años de edad registraron crecimientos en DAP de 10,4; 8,2 y
$5,8 \mathrm{~cm}$ y crecimientos en altura total de 4,$7 ; 4,3$ y 3,2 m para clases de sitio identificadas como I, II y III respectivamente.

\section{CONCLUSIONES}

El umbral inferior del incremento medio anual en diámetro y altura para $P$. alba en las plantaciones estudiadas resultó ser de 0,158 y 0,80 metros.

El umbral superior del incremento medio anual en diámetro y altura para $P$. alba en las plantaciones estudiadas resultó ser de 0,187 y 0,93 metros.

Las plantaciones de 4 y 5 años edad con espaciamiento $5 \times 5$ presentaron incrementos anuales en diámetro significativamente superiores a la plantación de 7 años de edad con espaciamiento $4 \times 4$.

La media ponderada de los crecimientos en diámetro y altura de $P$. alba para las plantaciones estudiadas es de 0,172 y 0,87 metros respectivamente y es una tendencia para plantaciones de edades similares en el centro oeste de la provincia del Chaco.

\section{REFERENCIAS}

Arteaga, B. y Castelán, L. 2008. Evaluación dasométrica temprana de una plantación agroforestal de tres especies introducidas, en el municipio de Huehuetla, Hidalgo. Revista Chapingo Serie Ciencias Forestales y del Ambiente 14(2):105-111.

Atanasio, M. 2014. Influencia de raleos selectivos sobre el crecimiento de $P$. alba. XXVIII Jornadas Forestales de Entre Ríos 2014. 8 p. 
Cuadra, D. 2012. La problemática forestal en la provincia del Chaco, Argentina. Un análisis desde la Geografía. Revista Geográfica Digital. IGUNNE. Facultad de Humanidades. UNNE. Resistencia, Chaco, Argentina. Año 9. No 18. 25 p.

Cuadra, D. 2011. Los recursos madereros Condiciones socioeconómicas vinculadas con su aprovechamiento en la provincia del Chaco. Revista Geográfica Digital. IGUNNE. Facultad de Humanidades. UNNE. Resistencia, Chaco., Argentina. Año 8. No 15. 15 p.

García, C., Parraguirre, C., y Rodríguez, B. 1992. Modelos de crecimiento para una plantación de caoba (Swietenia macrophylla King). Revista Ciencia Forestal en México, 17(71): 87-102.

Del Castillo, E y Tarnowski, C. (2006). Cultivo experimental de Prosopis chilensis (Molina) Stuntz., en el pedemonte de yungas. En: II Jornadas Forestales de Santiago del Estero, Argentina. 9 p.

Di Rienzo, J., Casanoves F., Balzarini M., Gonzalez L., Tablada M. y Robledo C. InfoStat versión 2013. Grupo InfoStat, FCA, Universidad Nacional de Córdoba, Argentina. URL http://www.infostat.com. ar [acceso noviembre 2013]

Dirección de Bosques. Ministerio de Economía, Industria y Empleo. Gobierno de la provincia del Chaco. http:// direcciondebosques.blogspot.com.ar/p/ estadisticas.html [acceso junio 2014]
Dirección de Producción Forestal. Ministerio de Agricultura, Ganadería y Pesca. Presidencia de la Nación. http://chacoforestal.blogspot.com.ar. [acceso junio 2014]

Ferrere, P., López, G.; Boca, R., Galetti, M. A., Esparrach C. y Pathauer, P. 2005. Efecto de la densidad de plantación sobre el crecimiento de Eucalyptus globulus en un ensayo Nelder modificado. Invest Agrar: Sist. Recur. For. 14(2):174-184.

Imaña, J. y Encinas, O. 2008. Epidometría forestal. Universidade de Brasilia, departamento de Engenharia Florestal. Universidad de Los Andes. Facultad de Ciencias Forestales. 72 p.

Ledesma, L., Barbona, S., Melgratti, M., Sayago, J., Margosa, L., Rodríguez, F., Gustin, A. V. y Enríquez, O. 1973. Introducción al Conocimiento de los Suelos del Chaco. Para el Programa de: Estudio y Extensión en Suelos de la provincia del Chaco.

Informe (de divulgación) de la Primera Etapa. Evaluación de los Factores Formadores del Suelo. Reconocimiento y Clasificación de suelos. Convenio entre el Instituto Nacional de Tecnología Agropecuaria (INTA) por intermedio de EEA INTA Sáenz Peña y el Gobierno de la Provincia del Chaco, Argentina. 157p.

Lifton, S. y Aguilar, E. 2007. Producción Forestal y Explotación del Monte en el Chaco. La Cadena Foresto Industrial en el Chaco. http:// www.coneschaco.org. ar [acceso junio 2014] 
Memorias del $1^{\circ}$ Congreso Forestal Chaco Sudamericano. Producción y Sustentabilidad. 10 p. ISSN 1853-5755.

Ministerio de Economía, Industria y Empleo. Gobierno de la provincia del Chaco. 2009. Plan de Competitividad. Conglomerado Productivo de Muebles de maderas del Chaco. http://www. mecon.gov.ar/programanortegrande/ docs/chaco_muebles_pc_resumen.pdf. 52p [acceso agosto 2014]

Morello, J. y Adámoli, J. 1974. La vegetación de la República Argentina. Las Grandes Unidades de Vegetación y Ambiente del Chaco Argentino. Secretaria de Estado de Agricultura y Ganadería de la nación. Instituto Nacional de Tecnología Agropecuaria. Instituto de Botánica Agrícola. Segunda parte: Vegetación y Ambiente de la provincia del Chaco. Serie Fitogeográfica № 13. 130 p.

Navall, M. y Senillani, M. 2004. Crecimiento y calidad maderera de plantaciones de Prosopis alba Gris., (algarrobo blanco) en el Área de Riego del Río Dulce, provincia de Santiago del Estero. XIX Jornadas Forestales de Entre Ríos. 6 p.

Patiño, F. 1995. El espaciamiento en plantaciones forestales. Revista Ciencia Forestal en México. 20(77):67-81.
Perez, V., Vicentini, G., Ocampo, L. y Vega, C. 2011. Evaluación preliminar de la calidad de sitio para Prosopis alba Griseb., mediante la utilización de criterios dasométricos. Memorias del $1^{\circ}$ Congreso Forestal Chaco Sudamericano. Producción y Sustentabilidad. 11 p. ISSN 1853-5755

Prokopiuk, D., Cruz, G., Grados, N., Garro, O. y Chiralt, A. 2000. Estudio comparativo entre frutos de Prosopis alba y Prosopis pallida. MULTEQUINA 9:35-45.

Ribaski, J. y Menezes, E. 2002. Disponibilidad y calidad del pasto buffel (Cenchrus ciliaris) en un sistema silvopastoril con algarrobo (Prosopis juliflora) en la región semiárida Brasileña. Revista Agroforestería en las Américas. 9(33):8-13.

Rojo, G., Mata, J., Angulo, X., Porras, C. y Velasquez, A. 2005. Modelos de índices de sitio para Hevea brasiliensis Müll. Arg. del clon IAN-710 en el norte de Chiapas. Revista de Sociedad, Cultura y Desarrollo sustentable. Ra Ximhai, Universidad Autónoma Indígena de México. 1(1):153-166.

Vicentini, G. y Pérez, V. 2011. Primeras evaluaciones de los efectos del suelo en forestaciones de Prosopis alba Griseb. del de la provincia de Formosa (Argentina). 